\title{
Incidence and costs of injuries to children and adults in the United States
}

\author{
Mark R. Zonfrillo ${ }^{1,4^{*}}$ D, Rebecca S. Spicer ${ }^{2}$, Bruce A. Lawrence ${ }^{2}$ and Ted R. Miller ${ }^{2,3}$
}

\begin{abstract}
Background: Injuries are a leading cause of death and acquired disability, and result in significant medical spending. Prior estimates of injury-related cost have been limited by older data, for certain population, or specific mechanisms.

Findings: This study estimated the incidence of hospital-treated nonfatal injuries in the United States (US) in 2013 and the related comprehensive costs. Injury-related emergency department (ED) visits and hospitalizations were identified using 2013 Healthcare Cost and Utilization Project (HCUP) data. Models estimated the costs of medical spending and lost future work due to injuries in 2013 U.S. dollars. A total of 31,038,072 nonfatal injury-related hospitalizations and ED visits were identified, representing 9.8 per 100 people. Hospital-treated nonfatal injuries cost an estimated \$1.853 trillion, including \$168 billion in medical spending, \$223 billion in work losses, and \$1.461 trillion in quality of life losses.

Conclusions: Approximately one in 10 individuals in the US is treated in the hospital for injury each year, with high corresponding costs. These data support priority-setting to reduce the injury burden in the US.
\end{abstract}

\section{Background}

Injuries are a leading cause of death and acquired disability in children and adults, and result in significant medical spending nationwide. Prior estimates of injury-related cost have been from much older data (Danseco et al. 2000; Miller et al. 2000; Corso et al. 2006), for certain populations (e.g., children (Miller et al. 2000; Zaloshnja et al. 2012; Roy et al. 2008), Medicaid recipients (Roy et al. 2008)), for certain mechanisms (e.g., consumer products (Lawrence and Miller, 2014), agricultural (Zaloshnja et al. 2012)), or for cost totals that only include medical care (Dieleman et al. 2016). This study provides contemporary national estimates of the incidence of hospital-treated nonfatal injuries for children and adults in the United States in 2013 and the related comprehensive costs by age, household income, payer, metropolitan residence, disposition, injury severity score, injury mechanism and injury intent. While incidence describes the magnitude of the problem, costs provide a better measure of burden by accounting for

\footnotetext{
* Correspondence: zonfrillo@brown.edu

${ }^{1}$ Hasbro Children's Hospital and Alpert Medical School of Brown University,

Providence, RI, USA

${ }^{4}$ Department of Emergency Medicine, Alpert Medical School at Brown

University, 55 Claverick St., 2nd floor, Providence, RI 02903, USA

Full list of author information is available at the end of the article
}

multiple injury consequences - e.g., severity, disability in a single unit of measurement.

\section{Methods}

Injury-related emergency department (ED) visits and hospitalizations were identified using 2013 Healthcare Cost and Utilization Project (HCUP) sample-based ED (Agency for Healthcare Research and Quality (AHRQ), n.d.) and inpatient (Agency for Healthcare Research and Quality (AHRQ)a, n.d.) datasets. This study used deidentified administrative data and was exempt from review by our institution. Injuries were defined as diagnoses 800-995 in the International Classification of Diseases, Ninth Revision, excluding late effects, 905-909. We used the standard external-cause matrix from the Centers for Disease Control and Prevention. (Centers for Disease Control and Prevention (CDC), n.d.-a; Centers for Disease Control and Prevention (CDC), n.d.-b).

We applied an established US injury cost model to the HCUP datasets to estimate the costs of injuries in 2013 U.S. dollars (Lawrence et al. 2015; Spicer et al. 2011). The Agency for Healthcare Research and Quality (AHRQ) weights HCUP Nationwide Emergency Department Sample (NEDS) and Nationwide Inpatient Sample (NIS) data to provide national estimates. 2014 NEDS 
estimates are based on sampling non-admitted cases from all EDs in 34 states. 2014 NIS estimates are based on a one-in-five random sample of inpatient discharges from every hospital in 44 states and the District of Columbia. (Agency for Healthcare Research and Quality
(AHRQ), n.d. Agency for Healthcare Research and Quality (AHRQ)a, n.d.).

Costs include medical spending and lost future work. In addition, we take a societal perspective by including the estimated value of lost quality of life. Medicalspending

Table 1 Cost of Hospital-Treated Nonfatal Injuries in the United States, 2013 (2013 United States \$)

\begin{tabular}{|c|c|c|c|c|c|c|}
\hline & Number & Medical & Work Loss & Quality of Life & Total Cost & Total Cost per Injury \\
\hline All & $31,038,072$ & $\$ 167,968,253,878$ & $\$ 223,116,101,911$ & $\$ 1,461,482,900,000$ & $\$ 1,852,567,255,789$ & $\$ 59,687$ \\
\hline \multicolumn{7}{|l|}{ Age Group } \\
\hline$<1$ & 261,308 & $\$ 1,432,470,125$ & $\$ 1,789,973,631$ & $\$ 22,287,154,385$ & $\$ 25,509,598,141$ & $\$ 97,623$ \\
\hline $1-11$ & $4,447,463$ & $\$ 12,032,484,055$ & $\$ 18,626,763,126$ & $\$ 181,322,230,105$ & $\$ 211,981,477,286$ & $\$ 47,663$ \\
\hline $12-17$ & $2,823,241$ & $\$ 8,816,169,603$ & $\$ 13,443,289,763$ & $\$ 145,256,948,411$ & $\$ 167,516,407,777$ & $\$ 59,335$ \\
\hline $18-24$ & $3,773,148$ & $\$ 13,600,427,953$ & $\$ 23,698,584,864$ & $\$ 186,399,030,940$ & $\$ 223,698,043,757$ & $\$ 59,287$ \\
\hline $25-34$ & $4,442,848$ & $\$ 17,457,020,563$ & $\$ 36,742,723,349$ & $\$ 205,840,515,715$ & $\$ 260,040,259,627$ & $\$ 58,530$ \\
\hline $35-44$ & $3,711,266$ & $\$ 15,005,365,402$ & $\$ 34,704,621,851$ & $\$ 150,463,641,515$ & $\$ 200,173,628,768$ & $\$ 53,937$ \\
\hline $45-64$ & $6,458,720$ & $\$ 40,319,428,609$ & $\$ 65,703,484,682$ & $\$ 314,280,411,223$ & $\$ 420,303,324,514$ & $\$ 65,075$ \\
\hline $65+$ & $4,800,513$ & $\$ 59,285,698,881$ & $\$ 28,387,430,398$ & $\$ 255,529,916,815$ & $\$ 343,203,046,094$ & $\$ 71,493$ \\
\hline \multicolumn{7}{|l|}{ Household Income Quartile } \\
\hline 0-25th percentile & $9,572,854$ & $\$ 47,498,738,599$ & $\$ 67,315,490,069$ & $\$ 419,419,736,702$ & $\$ 534,233,965,370$ & $\$ 55,807$ \\
\hline 26th-50th percentile & $8,347,113$ & $\$ 43,448,674,708$ & $\$ 57,933,681,503$ & $\$ 380,949,318,832$ & $\$ 482,331,675,043$ & $\$ 57,784$ \\
\hline 31st-75th percentile & $6,923,897$ & $\$ 39,294,070,461$ & $\$ 50,758,794,647$ & $\$ 339,582,090,153$ & $\$ 429,634,955,261$ & $\$ 62,051$ \\
\hline 76th-100th percentile & $5,533,774$ & $\$ 33,659,639,931$ & $\$ 40,997,146,789$ & $\$ 284,762,942,717$ & $\$ 359,419,729,437$ & $\$ 64,950$ \\
\hline \multicolumn{7}{|l|}{ Payer } \\
\hline Medicare & $5,599,892$ & $\$ 62,116,899,402$ & $\$ 40,517,276,172$ & $\$ 297,763,145,616$ & $\$ 400,397,321,190$ & $\$ 71,501$ \\
\hline Medicaid & $7,429,391$ & $\$ 29,171,568,219$ & $\$ 45,103,953,532$ & $\$ 314,054,136,094$ & $\$ 388,329,657,845$ & $\$ 52,269$ \\
\hline Other & $15,231,785$ & $\$ 64,317,973,204$ & $\$ 113,654,085,383$ & $\$ 712,228,646,085$ & $\$ 890,200,704,672$ & $\$ 58,444$ \\
\hline Private/Commercial/PPO/HMO & $2,776,540$ & $\$ 12,348,412,934$ & $\$ 23,823,408,746$ & $\$ 137,372,720,655$ & $\$ 173,544,542,335$ & $\$ 62,504$ \\
\hline \multicolumn{7}{|l|}{ Rural/Urban } \\
\hline Urban & $24,549,308$ & $\$ 134,880,605,547$ & $\$ 179,608,748,796$ & $\$ 1,164,443,500,000$ & $\$ 1,478,932,854,343$ & $\$ 60,243$ \\
\hline Rural & $6,292,866$ & $\$ 31,930,196,077$ & $\$ 41,617,835,131$ & $\$ 286,073,751,174$ & $\$ 359,621,782,382$ & $\$ 57,148$ \\
\hline \multicolumn{7}{|l|}{ Disposition } \\
\hline Treated and Released & $28,387,504$ & $\$ 66,949,530,984$ & $\$ 86,132,322,982$ & $\$ 788,923,246,256$ & $\$ 942,005,100,222$ & $\$ 33,184$ \\
\hline Admitted & $2,650,568$ & $\$ 101,018,722,894$ & $\$ 136,983,778,930$ & $\$ 672,559,644,444$ & $\$ 910,562,146,268$ & $\$ 343,535$ \\
\hline \multicolumn{7}{|l|}{ Injury Severity Score (ISS) } \\
\hline 0 & $4,007,518$ & $\$ 23,294,466,781$ & $\$ 9,224,703,223$ & $\$ 78,422,515,363$ & $\$ 110,941,685,367$ & $\$ 27,683$ \\
\hline$<5$ & $23,472,855$ & $\$ 81,231,767,072$ & $\$ 125,971,348,386$ & $\$ 797,810,907,004$ & $\$ 1,005,014,022,462$ & $\$ 42,816$ \\
\hline $5-14$ & $1,533,316$ & $\$ 31,204,880,868$ & $\$ 36,049,311,657$ & $\$ 287,923,572,318$ & $\$ 355,177,764,843$ & $\$ 231,640$ \\
\hline$\geq 15$ & $2,024,383$ & $\$ 32,237,139,158$ & $\$ 51,870,738,645$ & $\$ 297,325,896,016$ & $\$ 381,433,773,819$ & $\$ 188,420$ \\
\hline \multicolumn{7}{|l|}{ Region } \\
\hline Northeast & 486,635 & $\$ 18,095,855,894$ & $\$ 24,090,562,684$ & $\$ 119,160,902,927$ & $\$ 161,347,321,505$ & $\$ 331,557$ \\
\hline Midwest & 601,215 & $\$ 21,715,142,226$ & $\$ 29,756,502,527$ & $\$ 144,768,266,911$ & $\$ 196,239,911,664$ & $\$ 326,406$ \\
\hline South & $1,027,654$ & $\$ 36,344,852,910$ & $\$ 52,530,343,991$ & $\$ 258,276,688,480$ & $\$ 347,151,885,381$ & $\$ 337,810$ \\
\hline West & 535,064 & $\$ 24,862,871,864$ & $\$ 30,606,369,728$ & $\$ 150,353,786,126$ & $\$ 205,823,027,718$ & $\$ 384,670$ \\
\hline \multicolumn{7}{|l|}{ Hospital Type } \\
\hline Nonteaching & $11,451,866$ & $\$ 26,985,059,893$ & $\$ 34,347,299,866$ & $\$ 311,700,719,574$ & $\$ 373,033,079,333$ & $\$ 32,574$ \\
\hline Teaching & $11,244,316$ & $\$ 26,914,053,295$ & $\$ 35,054,247,432$ & $\$ 316,475,541,375$ & $\$ 378,443,842,102$ & $\$ 33,656$ \\
\hline
\end{tabular}


Table 2 Cost of Hospital-Treated Nonfatal Injuries in the United States by Mechanism, 2013 (2013 United States \$)

\begin{tabular}{|c|c|c|c|c|c|c|}
\hline & Number & Medical & Work Loss & Quality of Life & Total & Total Cost per Injury \\
\hline \multicolumn{7}{|l|}{ UNINTENTIONAL INJURIES } \\
\hline Cut/pierce & $1,912,149$ & $\$ 3,631,594,141$ & $\$ 4,922,277,919$ & $\$ 28,292,232,329$ & $\$ 36,846,104,389$ & $\$ 19,269$ \\
\hline Drowning/submersion & 9762 & $\$ 167,756,380$ & $\$ 585,909,962$ & $\$ 2,699,855,258$ & $\$ 3,453,521,600$ & $\$ 353,773$ \\
\hline Fall & $8,810,752$ & $\$ 64,201,479,065$ & $\$ 72,918,304,289$ & $\$ 505,230,956,245$ & $\$ 642,350,739,599$ & $\$ 72,905$ \\
\hline Fire/flame & 80,131 & $\$ 754,650,203$ & $\$ 728,172,630$ & $\$ 5,538,602,465$ & $\$ 7,021,425,298$ & $\$ 87,625$ \\
\hline Hot object/substance & 292,856 & $\$ 988,725,136$ & $\$ 1,708,949,240$ & $\$ 12,359,395,079$ & $\$ 15,057,069,455$ & $\$ 51,415$ \\
\hline Firearm & 35,858 & $\$ 358,159,858$ & $\$ 703,471,847$ & $\$ 2,983,640,338$ & $\$ 4,045,272,043$ & $\$ 112,815$ \\
\hline Machinery & 113,545 & $\$ 544,119,276$ & $\$ 1,846,062,780$ & $\$ 10,644,611,515$ & $\$ 13,034,793,571$ & $\$ 114,798$ \\
\hline MVT Occupant & $2,498,200$ & $\$ 13,763,057,426$ & $\$ 22,257,235,570$ & $\$ 97,781,307,804$ & $\$ 133,801,600,800$ & $\$ 53,559$ \\
\hline MVT Motorcyclist & 157,995 & $\$ 2,736,135,308$ & $\$ 5,580,436,675$ & $\$ 21,245,276,186$ & $\$ 29,561,848,169$ & $\$ 187,106$ \\
\hline MVT Pedal cyclist & 53,983 & $\$ 527,900,384$ & $\$ 1,123,476,662$ & $\$ 5,165,720,968$ & $\$ 6,817,098,014$ & $\$ 126,281$ \\
\hline MVT Pedestrian & 137,296 & $\$ 2,181,788,505$ & $\$ 3,548,142,901$ & $\$ 16,715,035,234$ & $\$ 22,444,966,640$ & $\$ 163,478$ \\
\hline MVT Unspecified & 219,970 & $\$ 1,053,152,143$ & $\$ 1,820,906,676$ & $\$ 9,972,036,013$ & $\$ 12,846,094,832$ & $\$ 58,399$ \\
\hline MVT Other & 17,891 & $\$ 111,877,667$ & $\$ 248,974,328$ & $\$ 1,464,181,060$ & $\$ 1,825,033,055$ & $\$ 102,010$ \\
\hline Pedal cyclist, other & 324,071 & $\$ 1,476,820,932$ & $\$ 3,263,738,075$ & $\$ 20,108,440,875$ & $\$ 24,848,999,882$ & $\$ 76,678$ \\
\hline Pedestrian, other & 19,026 & $\$ 169,750,289$ & $\$ 289,186,145$ & $\$ 1,884,173,134$ & $\$ 2,343,109,568$ & $\$ 123,153$ \\
\hline Transport, other & 294,317 & $\$ 2,358,186,236$ & $\$ 5,066,645,020$ & $\$ 29,023,314,277$ & $\$ 36,448,145,533$ & $\$ 123,840$ \\
\hline Bites and stings & $1,234,452$ & $\$ 2,547,309,358$ & $\$ 2,869,717,927$ & $\$ 27,803,209,134$ & $\$ 33,220,236,419$ & $\$ 26,911$ \\
\hline Other natural/environmental & 178,925 & $\$ 1,168,724,588$ & $\$ 1,414,612,181$ & $\$ 6,270,658,638$ & $\$ 8,853,995,407$ & $\$ 49,484$ \\
\hline Overexertion & $2,335,656$ & $\$ 6,555,552,120$ & $\$ 10,283,916,364$ & $\$ 65,455,667,267$ & $\$ 82,295,135,751$ & $\$ 35,234$ \\
\hline Poisoning & 469,441 & $\$ 2,957,904,882$ & $\$ 836,596,717$ & $\$ 19,797,098,590$ & $\$ 23,591,600,189$ & $\$ 50,255$ \\
\hline Struck by/against & $3,274,620$ & $\$ 8,646,126,741$ & $\$ 14,196,566,363$ & $\$ 142,802,749,122$ & $\$ 165,645,442,226$ & $\$ 50,585$ \\
\hline Suffocation & 37,714 & $\$ 2,035,124,771$ & $\$ 572,859,762$ & $\$ 2,216,460,767$ & $\$ 4,824,445,300$ & $\$ 127,921$ \\
\hline Other spec \& classification & $1,108,881$ & $\$ 4,457,686,594$ & $\$ 4,606,013,305$ & $\$ 54,204,054,262$ & $\$ 63,267,754,161$ & $\$ 57,055$ \\
\hline Other specified, NEC & 679,383 & $\$ 2,473,750,166$ & $\$ 3,703,018,649$ & $\$ 23,312,983,807$ & $\$ 29,489,752,622$ & $\$ 43,407$ \\
\hline Unspecified & $2,116,751$ & $\$ 9,391,792,223$ & $\$ 12,521,017,713$ & $\$ 60,497,266,532$ & $\$ 82,410,076,468$ & $\$ 38,932$ \\
\hline \multicolumn{7}{|l|}{ SELF-HARM INJURIES } \\
\hline Cut/pierce & 104,661 & $\$ 555,649,912$ & $\$ 1,526,402,750$ & $\$ 5,323,753,810$ & $\$ 7,405,806,472$ & $\$ 70,760$ \\
\hline Drowning/submersion & 265 & $\$ 3,418,777$ & $\$ 21,334,930$ & $\$ 76,901,789$ & $\$ 101,655,496$ & $\$ 383,493$ \\
\hline Fall & 2377 & $\$ 133,419,753$ & $\$ 216,937,041$ & $\$ 674,562,123$ & $\$ 1,024,918,917$ & $\$ 431,271$ \\
\hline Fire/flame & 1561 & $\$ 47,016,669$ & $\$ 35,395,270$ & $\$ 354,039,886$ & $\$ 436,451,826$ & $\$ 279,685$ \\
\hline Hot object/substance & 178 & $\$ 1,007,084$ & $\$ 2,947,776$ & $\$ 35,191,197$ & $\$ 39,146,057$ & $\$ 220,435$ \\
\hline Firearm & 2686 & $\$ 192,070,049$ & $\$ 336,463,687$ & $\$ 1,240,360,400$ & $\$ 1,768,894,136$ & $\$ 658,446$ \\
\hline MVT Other & 823 & $\$ 19,532,106$ & $\$ 42,591,826$ & $\$ 153,183,991$ & $\$ 215,307,923$ & $\$ 261,588$ \\
\hline Other natural/environmental & 123 & $\$ 2,690,121$ & $\$ 4,270,614$ & $\$ 13,651,834$ & $\$ 20,612,569$ & $\$ 167,359$ \\
\hline Poisoning & 279,444 & $\$ 2,061,419,009$ & $\$ 799,796,068$ & $\$ 9,522,661,850$ & $\$ 12,383,876,927$ & $\$ 44,316$ \\
\hline Suffocation & 9076 & $\$ 123,411,128$ & $\$ 873,402,854$ & $\$ 2,603,923,204$ & $\$ 3,600,737,186$ & $\$ 396,752$ \\
\hline Other specified \& classifiable & 1875 & $\$ 73,774,938$ & $\$ 172,400,608$ & $\$ 544,485,818$ & $\$ 790,661,364$ & $\$ 421,619$ \\
\hline Other specified, NEC & 27,623 & $\$ 175,461,682$ & $\$ 411,227,781$ & $\$ 1,413,948,899$ & $\$ 2,000,638,362$ & $\$ 72,426$ \\
\hline Unspecified & 7271 & $\$ 34,820,468$ & $\$ 79,108,294$ & $\$ 270,489,268$ & $\$ 384,418,030$ & $\$ 52,869$ \\
\hline \multicolumn{7}{|l|}{ ASSAULT } \\
\hline Cut/pierce & 73,932 & $\$ 491,701,003$ & $\$ 1,138,106,551$ & $\$ 5,476,377,890$ & $\$ 7,106,185,444$ & $\$ 96,118$ \\
\hline Drowning/submersion & 69 & $\$ 642,802$ & $\$ 1,964,335$ & $\$ 7,164,047$ & $\$ 9,771,184$ & $\$ 141,881$ \\
\hline Fall & 1646 & $\$ 12,022,160$ & $\$ 37,569,063$ & $\$ 169,630,025$ & $\$ 219,221,249$ & $\$ 133,196$ \\
\hline
\end{tabular}


Table 2 Cost of Hospital-Treated Nonfatal Injuries in the United States by Mechanism, 2013 (2013 United States \$) (Continued)

\begin{tabular}{|c|c|c|c|c|c|c|}
\hline & Number & Medical & Work Loss & Quality of Life & Total & Total Cost per Injury \\
\hline Fire/flame & 595 & $\$ 3,858,466$ & $\$ 9,261,217$ & $\$ 69,978,211$ & $\$ 83,097,894$ & $\$ 139,678$ \\
\hline Hot object/substance & 1962 & $\$ 15,993,005$ & $\$ 17,535,750$ & $\$ 153,556,467$ & $\$ 187,085,222$ & $\$ 95,368$ \\
\hline Firearm & 30,131 & $\$ 927,098,739$ & $\$ 1,530,254,784$ & $\$ 6,968,840,319$ & $\$ 9,426,193,842$ & $\$ 312,844$ \\
\hline MVT Occupant & 1660 & $\$ 6,798,022$ & $\$ 12,494,180$ & $\$ 130,515,813$ & $\$ 149,808,015$ & $\$ 90,225$ \\
\hline Poisoning & 1761 & $\$ 7,901,485$ & $\$ 2,498,250$ & $\$ 92,294,308$ & $\$ 102,694,043$ & $\$ 58,317$ \\
\hline Struck by/against & 611,218 & $\$ 2,512,923,350$ & $\$ 6,795,903,883$ & $\$ 56,416,139,291$ & $\$ 65,724,966,524$ & $\$ 107,531$ \\
\hline Suffocation & 3006 & $\$ 10,208,810$ & $\$ 27,416,530$ & $\$ 127,393,050$ & $\$ 165,018,390$ & $\$ 54,888$ \\
\hline Other specified \& classifiable & 119,372 & $\$ 604,801,671$ & $\$ 1,005,672,954$ & $\$ 7,496,925,042$ & $\$ 9,107,399,667$ & $\$ 76,294$ \\
\hline Other specified, NEC & 165,835 & $\$ 571,842,316$ & $\$ 1,014,664,619$ & $\$ 11,060,805,689$ & $\$ 12,647,312,624$ & $\$ 76,265$ \\
\hline Unspecified & 169,769 & $\$ 879,584,274$ & $\$ 2,260,749,692$ & $\$ 17,139,072,436$ & $\$ 20,279,406,402$ & $\$ 119,453$ \\
\hline \multicolumn{7}{|l|}{ UNDETERMINED INTENT } \\
\hline Cut/pierce & 3731 & $\$ 13,136,329$ & $\$ 31,468,294$ & $\$ 124,000,521$ & $\$ 168,605,144$ & $\$ 45,196$ \\
\hline Drowning/submersion & 233 & $\$ 912,250$ & $\$ 8,301,876$ & $\$ 31,576,211$ & $\$ 40,790,337$ & $\$ 175,358$ \\
\hline Fall & 3950 & $\$ 39,802,960$ & $\$ 99,196,652$ & $\$ 333,040,805$ & $\$ 472,040,417$ & $\$ 119,510$ \\
\hline Fire/flame & 2913 & $\$ 15,518,883$ & $\$ 31,234,477$ & $\$ 242,655,585$ & $\$ 289,408,944$ & $\$ 99,345$ \\
\hline Hot object/substance & 595 & $\$ 3,699,041$ & $\$ 7,221,322$ & $\$ 73,345,138$ & $\$ 84,265,501$ & $\$ 141,740$ \\
\hline Firearm & 4014 & $\$ 74,095,787$ & $\$ 129,846,857$ & $\$ 546,583,710$ & $\$ 750,526,354$ & $\$ 186,991$ \\
\hline MVT Other & 459 & $\$ 3,147,206$ & $\$ 6,780,905$ & $\$ 29,228,290$ & $\$ 39,156,401$ & $\$ 85,342$ \\
\hline Other natural/environmental & 2703 & $\$ 58,901,837$ & $\$ 45,949,242$ & $\$ 145,873,538$ & $\$ 250,724,617$ & $\$ 92,761$ \\
\hline Poisoning & 126,707 & $\$ 892,799,765$ & $\$ 326,704,590$ & $\$ 5,843,044,484$ & $\$ 7,062,548,839$ & $\$ 55,739$ \\
\hline Suffocation & 267 & $\$ 1,684,991$ & $\$ 9,729,591$ & $\$ 19,947,053$ & $\$ 31,361,635$ & $\$ 117,601$ \\
\hline Other specified \& classifiable & 463 & $\$ 4,000,582$ & $\$ 9,817,663$ & $\$ 43,121,482$ & $\$ 56,939,727$ & $\$ 122,885$ \\
\hline Other specified, NEC & 14,025 & $\$ 64,785,622$ & $\$ 98,439,086$ & $\$ 539,613,549$ & $\$ 702,838,257$ & $\$ 50,114$ \\
\hline Unspecified & 76,146 & $\$ 313,398,577$ & $\$ 320,997,827$ & $\$ 2,566,738,535$ & $\$ 3,201,134,939$ & $\$ 42,039$ \\
\hline \multicolumn{7}{|l|}{ LEGAL INTERVENTION ${ }^{a}$} \\
\hline Cut/pierce & 1449 & $\$ 3,102,948$ & $\$ 6,635,734$ & $\$ 22,866,321$ & $\$ 32,605,003$ & $\$ 22,502$ \\
\hline Firearm & 1383 & $\$ 41,617,599$ & $\$ 57,218,311$ & $\$ 234,236,902$ & $\$ 333,072,812$ & $\$ 240,832$ \\
\hline Poisoning & 349 & $\$ 645,957$ & $\$ 336,617$ & $\$ 13,470,021$ & $\$ 14,452,595$ & $\$ 41,469$ \\
\hline Struck by/against & 41,824 & $\$ 127,374,328$ & $\$ 266,063,345$ & $\$ 1,325,708,220$ & $\$ 1,719,145,893$ & $\$ 41,104$ \\
\hline Other specified \& classifiable & 1174 & $\$ 9,406,164$ & $\$ 8,221,940$ & $\$ 41,502,028$ & $\$ 59,130,132$ & $\$ 50,375$ \\
\hline Other specified, NEC & 121 & $\$ 1,064,568$ & $\$ 2,915,549$ & $\$ 9,994,667$ & $\$ 13,974,784$ & $\$ 115,142$ \\
\hline Unspecified & 6937 & $\$ 20,238,437$ & $\$ 42,810,948$ & $\$ 214,315,994$ & $\$ 277,365,380$ & $\$ 39,984$ \\
\hline \multicolumn{7}{|l|}{ UNSPECIFIED INTENT } \\
\hline Drowning/submersion & 443 & $\$ 27,326,952$ & $\$ 54,731,485$ & $\$ 204,710,316$ & $\$ 286,768,754$ & $\$ 646,970$ \\
\hline Other natural/environmental & 49,694 & $\$ 686,083,672$ & $\$ 522,349,852$ & $\$ 2,757,831,073$ & $\$ 3,966,264,597$ & $\$ 79,814$ \\
\hline Poisoning & 81,013 & $\$ 677,091,885$ & $\$ 163,997,422$ & $\$ 4,002,093,054$ & $\$ 4,843,182,361$ & $\$ 59,783$ \\
\hline Suffocation & 28,327 & $\$ 1,760,326,274$ & $\$ 379,312,268$ & $\$ 1,302,485,774$ & $\$ 3,442,124,316$ & $\$ 121,514$ \\
\hline Other specified \& classifiable & 57,020 & $\$ 351,856,081$ & $\$ 246,844,436$ & $\$ 698,824,829$ & $\$ 1,297,525,346$ & $\$ 22,756$ \\
\hline Unspecified & $2,499,592$ & $\$ 18,048,042,991$ & $\$ 24,246,394,615$ & $\$ 139,111,309,043$ & $\$ 181,405,746,649$ & $\$ 72,574$ \\
\hline
\end{tabular}

MVT Motor vehicle traffic

NEC Not elsewhere classifiable

*Deaths due to injuries inflicted by police or other law enforcement agents 
includes hospital and professional services, emergency transport, rehabilitation, prescriptions, home health care, and medical equipment. Lost future work includes future value of work that patients would be unable to do if they were killed or permanently disabled. Future costs were stated in present value using a $3 \%$ discount rate. (Lawrence and Miller 2014). We valued quality of life lost with a widely published value of $\$ 152,733$ per quality-adjusted life year, net of work loss (Miller and Hendrie, 2013). We used the same value per QALY of present-value life expectancy for all people. Because life-expectancy varies by age and sex, the total QALY loss to a death or permanently disabling injury also does.

\section{Results}

\section{Incidence}

A total of 31,038,072 nonfatal injury-related hospitalizations and ED visits were identified in 2013 (Table 1). This represents 9.8 per 100 people.

\section{Costs}

Hospital-treated nonfatal injuries in 2013 cost an estimated \$1.853 trillion, including \$168 billion in medical spending, \$223 billion in work losses, and quality of life losses valued at $\$ 1.461$ trillion (Table 1). The total estimated cost per injury was approximately $\$ 59,700$, including approximately $\$ 5400$ in medical spending, $\$ 7200$ in lost future work and $\$ 47,100$ in quality of life losses. The total costs per injury were highest for the oldest and youngest age groups; individuals $<1$ year old $(\$ 97,623)$ and 65 years and older $(\$ 71,493)$. Total cost per injury was slightly higher for those with Medicare and $\mathrm{Me}$ dicaid versus those with commercial insurance or other payer types. While $91.5 \%$ of patients with injuries were discharged, this represented only $8.8 \%$ of costs. In contrast, the $8.5 \%$ of patients admitted represented $91.2 \%$ of costs.

Falls and struck by/against injuries contributed to 35\% of nonfatal injury costs (Table 2) and were the leading causes in all age groups (results not shown). The most severe and debilitating injuries will result in higher costs. Among hospital-treated nonfatal injuries, near-drownings, self-harm, and firearm-related violence are the most costly. The external cause of injury was not coded for cases accounting for $9 \%$ of total injury costs.

\section{Discussion}

Approximately one in 10 individuals in the United States is injured each year are treated in a hospital, with high corresponding costs. Our calculated rate of 9.8 injuries per 100 people in 2013 is nearly half that of the 18.135 injuries per 100 population estimate by Corso et al. based on data from 2000. (Corso et al. 2006) While a prior study of US spending on personal health care found that injuries accounted for $\$ 168$ billion (8\%) of the $\$ 2.1$ trillion in medical spending in 2013, our comprehensive injury medical spending estimate exceeds that, even while excluding the 12 million injuries treated in physician's offices and clinics. (Dieleman et al. 2016) Limitations of the study include the absence of outpatient and ambulatory data, as well as the possibility of misclassification based on ICD codes.

These cost data from 2013 support priority-setting and selection of interventions to reduce the burden of injury in the United States.

\section{Abbreviations}

ED: Emergency department; HCUP: Healthcare Cost and Utilization Project; MVT: Motor vehicle traffic; NEC: Not otherwise classifiable

\section{Funding}

Dr. Zonfrillo was supported by the National Institutes of Health, Eunice Kennedy Shriver National Institute of Child Health and Human Development, grant 5K08HD073241.

Availability of data and materials

Please contact author for data requests.

Authors' contributions

This study was designed by MRZ, RSS, and TRM. Data analysis and collection was done, or supervised, by MRZ, RSS, BAL, TRM. Data interpretation and manuscript preparation were done by MRZ, RSS, BAL, TRM. All authors made significant contributions to drafting and critical revision of the manuscript and approved the final version.

Ethics approval and consent to participate

The study was not human subjects research and was exempt from review. Consent to participate not applicable.

\section{Competing interests}

The authors declare that they have no competing interests.

\section{Publisher's Note}

Springer Nature remains neutral with regard to jurisdictional claims in published maps and institutional affiliations.

\section{Author details}

${ }^{1}$ Hasbro Children's Hospital and Alpert Medical School of Brown University, Providence, RI, USA. ²Pacific Institute for Research and Evaluation, Calverton, MD, USA. ${ }^{3}$ Curtin University School of Public Health, Perth, Australia. ${ }^{4}$ Department of Emergency Medicine, Alpert Medical School at Brown University, 55 Claverick St., 2nd floor, Providence, RI 02903, USA.

Received: 12 June 2018 Accepted: 14 September 2018

Published online: 08 October 2018

\footnotetext{
References

Agency for Healthcare Research and Quality (AHRQ). Nationwide Emergency Department Sample (NEDS). Healthcare Cost and Utilization project (HCUP). http://www.hcup-us.ahrq.gov/nedsoverview.jsp. Accessed 15 Nov 2017. Agency for Healthcare Research and Quality (AHRQ)a. Nationwide Inpatient Sample (NIS). Healthcare Cost and Utilization project (HCUP). http://www. hcup-us.ahrq.gov/nisoverview.jsp. Accessed 15 Nov 2017.

Centers for Disease Control and Prevention (CDC). National Center for Health Statistics: ICD-9 Framework for presenting injury mortality data. https://www. cdc.gov/nchs/injury/ice/matrix.htm. Accessed 15 Nov 2017.

Centers for Disease Control and Prevention (CDC). External cause-of-injury mortality matrix based on ICD-9 external cause of injury codes. https://www. cdc.gov/nchs/injury/ice/matrix.htm. Accessed15 Nov 2017.

Corso P, Finkelstein E, Miller T, Fiebelkorn I, Zaloshnja E. Incidence and lifetime costs of injuries in the United States. Inj Prev. 2006 Aug;12(4):212-8.
} 
Danseco ER, Miller TR, Spicer RS. Incidence and costs of 1987-1994 childhood injuries: demographic breakdowns. Pediatrics. 2000 Feb;105(2):E27.

Dieleman JL, Baral R, Birger M, et al. US spending on personal health care and public health, 1996-2013. JAMA. 2016:316(24):2627-46.

Lawrence BA, Miller TR. Medical and work loss cost estimation methods for the WISQARS cost of injury module, final report to the Centers for Disease Control and Prevention. 2014. http://www.pire.org/documents/ WisqarsCostMethods.pdf. Accessed 15 Nov 2017.

Lawrence BA, Spicer RS, Miller TR. A fresh look at the costs of non-fatal consumer product injuries. Inj Prev. 2015;21(1):23-9. https://doi.org/10.1136/injuryprev2014-041220 Epub 2014 Aug 1.

Miller TR, Hendrie D. Economic evaluation of public health Laws and their enforcement. In: A Wagenaar AC, Burris S, editors. Public health law research: theory and methods. San Francisco, CA: Jossey-Bass; 2013. p. 347-78.

Miller TR, Romano EO, Spicer RS. The cost of childhood unintentional injuries and the value of prevention. Future child. Spring-Summer. 2000;10(1):137-63.

Roy S, Smith M, Coben J, Helmkamp J. Utilization and costs of injury-related acute care services among children and adolescents in a state Medicaid program. Matern Child Health J. 2008;12(5):641-9 Epub 2007 Oct 24.

Spicer RS, Miller TR, Hendrie D, Blincoe LJ. Quality-adjusted life years lost to road crash injury: updating the injury impairment index. Ann Adv Automot Med. 2011:55:365-77.

Zaloshnja E, Miller TR, Lawrence B. Incidence and cost of injury among youth in agricultural settings, United States, 2001-2006. Pediatrics. 2012;129(4):728-34. https://doi.org/10.1542/peds.2011-2512 Epub 2012 Mar 12.

\section{Submit your manuscript to a SpringerOpen ${ }^{\circ}$ journal and benefit from:}

- Convenient online submission

- Rigorous peer review

- Open access: articles freely available online

High visibility within the field

- Retaining the copyright to your article

Submit your next manuscript at $\boldsymbol{\nabla}$ springeropen.com 